\title{
Effect of piroxicam on lipid membranes : Drug encapsulation and gastric toxicity aspects
}

\section{Wilkosz, Natalia}

2017-03-30

Wilkosz , N , Rissanen , S , Cyza , M , Szybka , R , Nowakowska, M , Bunker , A, Rog , T \& Kepczynski , M 2017 , ' Effect of piroxicam on lipid membranes : Drug encapsulation and gastric toxicity aspects ' , European Journal of Pharmaceutical Sciences, vol. 100 , pp. 116-125 . https://doi.org/10.1016/j.ejps.2017.01.007

http://hdl.handle.net/10138/311787

https://doi.org/10.1016/j.ejps.2017.01.007

cc_by_nc_nd

acceptedVersion

Downloaded from Helda, University of Helsinki institutional repository.

This is an electronic reprint of the original article.

This reprint may differ from the original in pagination and typographic detail.

Please cite the original version. 


\section{Effect of piroxicam on lipid membranes: Drug} encapsulation and gastric toxicity aspects

Natalia Wilkosz ${ }^{\mathrm{a}, \sharp}$, Sami Rissanen $\stackrel{\mathrm{b}, \sharp}{ }$, Małgorzata Cyza ${ }^{\mathrm{a}}$, Renata Szybka ${ }^{\mathrm{a}}$, Maria Nowakowska ${ }^{\mathrm{a}}$, Alex Bunkerc, Tomasz Róg ${ }^{\mathrm{b}, \mathrm{d}, *}$ and Mariusz Kepczynski ${ }^{\mathrm{a}, *}$

${ }^{a}$ Faculty of Chemistry, Jagiellonian University, Ingardena 3, 30-060 Kraków, Poland

${ }^{\mathrm{b}}$ Department of Physics, Tampere University of Technology, PO Box 692, FI-33101 Tampere, Finland

${ }^{c}$ Centre for Drug Research, Division of Pharmaceutical Biosciences, Faculty of Pharmacy, University of Helsinki, Helsinki 00100, Finland

${ }^{\mathrm{d}}$ Department of Physics, University of Helsinki, Gustaf Hällströmin katu 2a, FI-00560 Helsinki, Finland.

$\$$ These authors equally contributed to the work. 
Wilkosz et al. European Journal of Pharmaceutical Sciences 2017, 100, 116-125. http://dx.doi.org/10.1016/j.ejps.2017.01.007

\section{Corresponding Authors}

Mariusz Kepczynski

Faculty of Chemistry, Jagiellonian University,

Ingardena 3, 30-060 Kraków, Poland

*Tel +48 12 6632020; Fax +48 12 6340515; e-mail kepczyns@chemia.uj.edu.pl (M.K.).

*Tel +358 40198 1010; Fax +358 33115 3015; e-mail tomasz.rog@gmail.com (T.R.). 
Wilkosz et al. European Journal of Pharmaceutical Sciences 2017, 100, 116-125. http://dx.doi.org/10.1016/j.ejps.2017.01.007

\section{ABSTRACT}

Uptake of piroxicam, a non-steroidal anti-inflammatory drug, from the intestines after oral intake is limited due to its low solubility and its wide use is associated with several side effects related to the gastrointestinal tract. In this study, all-atom molecular dynamics (MD) simulations and fluorescent spectroscopy were employed to investigate the interaction of piroxicam in neutral, zwitterionic, and cationic forms with lipid bilayers composed of phosphatidylcholine, cholesterol, and PEGylated lipids. Our study was aimed to assess the potential for encapsulation of piroxicam in liposomal carriers and to shed more light on the process of gastrointestinal tract injury by the drug. Through both the MD simulations and laser scanning confocal microscopy, we have demonstrated that all forms of piroxicam can associate with the lipid bilayers and locate close to the water-membrane interface. Conventional liposomes used in drug delivery are usually stabilized by the addition of cholesterol and have their bloodstream lifetime extended through the inclusion of PEGylated lipids in the formulation to create a protective polymer corona. For this reason, we tested the effect of these two modifications on the behavior of piroxicam in the membrane. When the bilayer was PEGylated, piroxicam localize to the PEG layer and within the lipid headgroup region. This suggests that PEGylated liposomes are capable of carrying a larger quantity of piroxicam than the conventional ones.

Keywords Piroxicam; NSAIDs; lipid bilayer; PEGylated liposomes; gastric toxicity; MD simulations 
Wilkosz et al. European Journal of Pharmaceutical Sciences 2017, 100, 116-125. http://dx.doi.org/10.1016/j.ejps.2017.01.007

Chemical compounds studied in this article:

Piroxicam (PubChem CID: 54676228); 1-Palmitoyl-2-oleoyl-sn-glycero-3-phosphocholine (PubChem CID: 5497103); Cholesterol (PubChem CID: 5997; N-(Carbonylmethoxypoly(ethylene glycol) 2000)-1,2-distearoyl-sn-glycero-3-phosphoethanolamine, sodium salt (PubChem CID: 86278269) 
Wilkosz et al. European Journal of Pharmaceutical Sciences 2017, 100, 116-125. http://dx.doi.org/10.1016/j.ejps.2017.01.007

\section{Introduction}

Liposomes are approximately spherical vesicles, in which one (unilamellar) or more (multilamellar) concentric lipid membranes enclose an aqueous phase. Liposomal bilayers can be seen as simplified models of biological membranes and are used in this capacity as tools to study the passive uptake of drugs (Kepczynski et al., 2008; van Balen et al., 2004). In addition, since the 1970's, liposomes have been used as drug carriers in a number of clinical applications, for example in cancer chemotherapy and the treatment of fungal infections (Lasic, 1998). A significant problem that is encountered in the development of liposome-based drug delivery systems is their low stability, especially in the presence of serum ingredients. In many cases liposomes were found to lose most of their content immediately after contact with blood serum (Wytrwal et al., 2014). Moreover, serum proteins bind or insert into the lipid bilayer causing its destabilization and, in some cases, play a role in complement activation that marks the liposomes for removal by the mononuclear phagocyte system (MPS) (Maurer et al., 2001).

The main strategies proposed to improve the stability of lipid vesicles and to obtain longcirculating liposomes involve (i) optimizing the lipid composition of the membrane, for example the incorporation of cholesterol (Chol) reduces the binding/insertion of blood proteins thus increasing considerably the stability of liposomes (Maurer et al., 2001); (ii) inclusion of lipids with their headgroups functionalized with a polymer to form a corona around the liposome ("sterically stabilized" liposome, SSL) that somehow impedes uptake by the MPS. Poly(ethylene glycol) (PEG) is the current gold standard for the protective polymer corona and liposomes containing the PEG corona are referred to as being "PEGylated" (Tarcholin, 2005). In previous work, we have demonstrated that PEGylation significantly increases the ability of liposomes to accumulate hydrophobic compounds (Kepczynski et al., 2008). Recently, using experimental 
Wilkosz et al. European Journal of Pharmaceutical Sciences 2017, 100, 116-125. http://dx.doi.org/10.1016/j.ejps.2017.01.007

methods and all-atom molecular dynamics (MD) simulations we demonstrated that the presence of the PEG corona around liposomes serves as an additional volume, where the hydrophobic compounds can be accumulated, thus improving the drug-loading efficiency (Dzieciuch et al., 2015).

The main objective of this paper is to investigate the interactions between an ionizable waterinsoluble drug and the conventional and PEGylated membrane. Piroxicam (4-hydroxy-2-methyl3-(pyrid-2-yl-carbamoyl)-2H-1,2-benzothiazine 1,1-dioxide, Px) was chosen as a model drug. The Px molecule is a well-known example of a Biopharmaceutics Classification System Class II drug, with low solubility and high permeability, for which oral absorption is considered to be dissolution-rate limited. For this reason, Px is used as a model drug in many studies focused on developing new formulations with enhanced dissolution kinetics. Px is a non-steroidal antiinflammatory drug (NSAID) belonging to the oxicam class (Banerjee and Sarkar, 2002).

NSAIDs are among the most commonly used drugs in the world due to their antipyretic, analgesic and anti-inflammatory properties (Pereira-Leite et al., 2013). They can be used to relieve pain caused, inter alia, by injury, arthritis, menstrual cramps and musculoskeletal conditions (Ferreira de Souza et al., 2010; Shojaee et al., 2013). Px, in particular, is known for its analgesic and anti-inflammatory efficacy. However, like other NSAIDs, Px causes many side effects. These include heart attack, stroke, and bleeding from the stomach and intestines (Shojaee et al., 2013). A large number of side effects of oral administration of Px and its hydrophobic nature are factors that limit its application. One way to overcome these problems is the use of liposomes as drug carriers. It was observed that liposomal encapsulation of drugs can considerably change their pharmacokinetics and biodistribution, focusing delivery to the target site, thus reducing their toxicity (Nawalany et al., 2009). In addition, it was shown that the 
Wilkosz et al. European Journal of Pharmaceutical Sciences 2017, 100, 116-125. http://dx.doi.org/10.1016/j.ejps.2017.01.007

encapsulation of piroxicam within liposomes increases the topical anti-inflammatory effect (Canto et al., 1999), reduces cytotoxicity, and enhances anti-inflammatory responses in vitro (Chiong et al., 2013).

In this article, we present the results of a combined in silico and in vitro study on the interactions between Px and model membranes of both conventional and PEGylated liposomes. The evaluation of the $\mathrm{Px}$ - membrane interaction is very important to predict: (i) the distribution of the drug in the body and ultimately to evaluate both the therapeutic effect and toxicity, and (ii) the potential for encapsulation of the drug in liposomal carriers. Only the neutral form of oxicams is suggested to be biologically active, and in particular it can cross the blood-brain barrier (Jolliet et al., 1997; Tsai et al., 1993). Therefore, in this work we have focused on two neutral forms of the drug. Moreover, because orally administered Px causes gastric side-effects (ulceration, bleeding and formation of holes in the stomach), we included its cationic form (present under highly acidic conditions) in this study. In silico molecular modeling provides an effective "computational microscope" on the system in question, revealing a level of detail that is often very difficult or even impossible to achieve experimentally (Lee et al., 2009). We used allatom MD simulations to estimate location and orientation of the drug inside the lipid bilayer and its effect on the membrane properties. Next, we applied a spectroscopic titration method to determine the affinity of Px for conventional and PEGylated membranes. The systems studied in this paper can serve as models for other ionizable drugs interacting with biomembranes and the results can be generalized to describe the behavior of other small organic molecules, applied as drugs, both in passive drug uptake and in liposome-based drug delivery.

\section{Materials and Methods}


Wilkosz et al. European Journal of Pharmaceutical Sciences 2017, 100, 116-125. http://dx.doi.org/10.1016/j.ejps.2017.01.007

\subsection{Materials}

Piroxicam (Px, meets USP testing specifications) and 1-palmitoyl-2-oleoyl-sn-glycero-3phosphocholine (POPC, $\geq 99 \%$ ) were purchased from Sigma and used as received. $N$-(Carbonylmethoxypoly(ethylene glycol) 2000)-1,2-distearoyl-sn-glycero-3-phosphoethanolamine sodium salt (DSPE-PEG) was received from NOF Europe (Belgium) NV. All solvents were obtained from Aldrich and were of spectroscopic grade. For all subsequent studies the DMF stock solution of the drug $\left(c_{\mathrm{Px}}=3.3 \mathrm{mM}\right)$ was prepared and used. All experiments were conducted under phosphate-buffered saline (PBS) conditions, at $\mathrm{pH}$ 4.0. Millipore-quality water was used in the experiments.

\subsection{Apparatus}

UV-vis absorption spectra of samples were measured at room temperature using a Varian Cary 50 spectrophotometer. Steady-state fluorescence spectra were recorded on an SLM-AMINCO 8100 spectrofluorometer at room temperature. Emission spectra were corrected for the wavelength dependence of the detector response by using an internal correction function provided by the manufacturer.

\subsection{Preparation of Liposomes}

To prepare small unilamellar liposomes (SUVs), POPC was weighed into a vial and dissolved in a small volume of chloroform. A thin film of lipid was formed by evaporating the solvent under a gentle stream of nitrogen. In the case of PEGylated liposomes, $7 \mathrm{~mol} \%$ of DSPE-PEG dissolved in ethanol was added to the vial before the evaporation. The dry film was hydrated with the buffer to achieve the lipid concentration of 1.0 or $2.5 \mathrm{mg} / \mathrm{mL}$ and vortex mixed for 2 min. The lipid dispersion was subjected to five freeze-thaw cycles from liquid nitrogen temperature to the temperature of $60{ }^{\circ} \mathrm{C}$. SUVs were formed by sonication for $10 \mathrm{~min}$ in an ice 
Wilkosz et al. European Journal of Pharmaceutical Sciences 2017, 100, 116-125. http://dx.doi.org/10.1016/j.ejps.2017.01.007

bath using a titanium tip SONICS VC 130 sonicator. The SUV dispersion was centrifuged using a MPW-250 Medical Instrument centrifuge for $5 \mathrm{~min}$ at 13,000 rpm to remove titanium particles coming from the sonicator horn.

For visualization with laser scanning confocal microscopy (LSCM), giant unilamellar liposomes (GUVs) were prepared by electroformation as follows. ITO-glasses and a Teflon spacer were washed in a sequence of water, ethanol, chloroform, ethanol, and water. Lipid was dissolved in chloroform and this solution was spread on the conductive surface of both ITOcoated glasses with a syringe. The glasses were dried in a vacuum desiccator for 2 hours. Two ITO-coverslips and the spacer were then assembled into a cell. The cell was filled with a 300$\mathrm{mM}$ sucrose solution. The ITO-glasses were connected to a pulse generator and an alternating voltage $(U=0.5 \mathrm{~V}, f=10 \mathrm{~Hz})$ was applied. The voltage was increased every 10 minutes by 0.5 $\mathrm{V}$ until the final value of $2 \mathrm{~V}$ was reached. This voltage was maintained for 1.5 hours and then it was slowly returned to $0 \mathrm{~V}$. The final lipid concentration was $2.5 \mathrm{mg} / \mathrm{mL}$.

\subsection{Determination of Liposome Binding Constant}

The binding constant $\left(K_{\mathrm{b}}\right)$ of Px to a lipid bilayer was determined using a spectroscopic titration method that was described in greater detail in our previous work (Kępczyński et al., 2002). Briefly, the sets of steady-state emission spectra of the equilibrated aqueous solutions containing the constant concentration of $\mathrm{Px}\left(c_{\mathrm{Px}}=32.9 \mu \mathrm{M}\right)$ and various concentrations of lipid were measured. The experiments were repeated at least three times. The incubation time required to reach equilibrium was determined prior to the experiments. The fluorescence intensity of the dye increased upon its partitioning into the lipid bilayer. This increase fulfilled the relation

$$
F=\frac{F_{\text {init }}+F_{\text {comp }} K_{\mathrm{b}}[\mathrm{L}]}{1+K_{\mathrm{b}}[\mathrm{L}]},
$$


Wilkosz et al. European Journal of Pharmaceutical Sciences 2017, 100, 116-125. http://dx.doi.org/10.1016/j.ejps.2017.01.007

where $F_{\text {init }}$ and $F$ are the fluorescence intensities measured in the absence of lipid and at the lipid concentration of [L], respectively. $F_{\text {comp }}$ is the asymptotic value of the fluorescence intensity at complete binding. To obtain $K_{\mathrm{b}}, F$ versus [L] data were plotted and fitted to equation 1 using a nonlinear regression routine. In this study, $K_{\mathrm{b}}$ was given in units of $(\mathrm{mg} / \mathrm{mL})^{-1}$.

\subsection{Laser scanning confocal microscopy (LSCM)}

Ten microliters of the solution containing GUVs treated with Px for $2 \mathrm{~h}$ were applied onto a microscope slide and covered with a cover glass. Visualization of the liposomes was performed using an A1-Si Nikon Inc. (Japan) confocal laser scanning system built on an inverted microscope Nikon Ti-E(Japan). A Plan Apo 100×/1.4 Oil DIC objective and 405 and $488 \mathrm{~nm}$ diode lasers was used in all cases.

\section{MD simulations}

A summary of the systems studied is listed in Table 1. We performed atomistic MD simulations for model systems containing: (1) Lipid bilayers composed of POPC (systems M1) and (2) POPC bilayer with $20 \mathrm{~mol} \%$ of cholesterol (the POPC/Chol mixed membrane, systems M2). Both systems were hydrated with 8000 water molecules ( $\sim 62$ water molecules per lipid) and included four Px molecules initially placed at different locations. In systems M1a and M2a all Px molecules were located in bulk water (Figure S1A and C), whereas systems M1b and M2b contained one Px molecule in the aqueous phase, and the remaining within the hydrophobic region of the membrane (Figure 1A and C); (3) POPC bilayer with $\sim 7 \mathrm{~mol} \%$ of DSPE-PEG and 12 molecules of Px placed at different initial positions (Figure 1E). $\mathrm{Na}^{+}$and $\mathrm{Cl}^{-}$ions were added in order to achieve physiological salt concentration $(150 \mathrm{mM})$. 
Wilkosz et al. European Journal of Pharmaceutical Sciences 2017, 100, 116-125. http://dx.doi.org/10.1016/j.ejps.2017.01.007

It has been shown that the neutral form of Px can exist in 7 different tautomers (Ivanova et al., 2015). Due to computational limitation, we considered only two tautomers: the most stable enolamide tautomer (referred to as PxA) and keto-amide tautomer (referred to as PxE). We also calculated the zwitterionic form (PxZw) and the cationic form $\left(\mathrm{Px}^{+}\right)$. The chemical structures of all forms of Px we studied are illustrated in Scheme 1.

Table 1. Summary of the simulated systems. For each system, the table indicates the number of molecules in the given system, the simulation time and the number of repeats. All the systems were simulated for all four Px forms.

\begin{tabular}{cccccccc}
\hline System & Px & POPC & CHOL & DSPE-PEG & Water & $\mathrm{Na}^{+} / \mathrm{Cl}^{-}$ & $\begin{array}{c}\text { Simulation } \\
\text { length }(\mathrm{ns})\end{array}$ \\
\hline M1a & $4^{a}$ & 128 & - & - & 8000 & $22 / 22$ & $2 \times 300$ \\
M1b & $4^{b}$ & 128 & - & - & 8000 & $22 / 22$ & $1 \times 300$ \\
M2a & $4^{a}$ & 102 & 26 & - & 8000 & $22 / 22$ & $2 \times 300$ \\
M2b & $4^{b}$ & 102 & 26 & - & 8000 & $22 / 22$ & $1 \times 300$ \\
M3 & 12 & 464 & - & 36 & 61000 & $136 / 100$ & $1 \times 500$ \\
\hline
\end{tabular}

${ }^{a} \overline{\text { Four molecules of Px were placed in the aqueous phase. }{ }^{b} \text { One molecule of Px was placed in the }}$ aqueous phase and three molecules were placed in the hydrophobic core of the membrane.

Simulations were carried out using the GROMACS 4.6 software package (Hess et al., 2008). To parameterize all lipid molecules and forms of Px, the all-atom OPLS force field was employed (Jorgensen et al., 1996) with a recent extension for lipids (Maciejewski et al., 2014; Kulig et al., 2015; Kulig et al., 2016). Partial charges for the PxA, PxE, PxZw, and $\mathrm{Px}^{+}$forms were derived according to the OPLS methodology; details regarding all calculations can be found in a previous publication (Li et al., 2012). The TIP3P model, compatible with the OPLS- 
Wilkosz et al. European Journal of Pharmaceutical Sciences 2017, 100, 116-125. http://dx.doi.org/10.1016/j.ejps.2017.01.007

AA force field, was used for water (Jorgensen et al., 1983). The time step was set to $2 \mathrm{fs}$, and the simulations were carried out at 1 bar and $300 \mathrm{~K}$. The Nosé-Hoover method was used to couple the temperature with separate heat baths for the membrane and the rest of the system with time constants of 0.4 ps (Nose, 1984; Hoover, 1985). The reference pressure was maintained using the semi-isotropic Parrinello-Rahman barostat (Parrinello and Rahman, 1981). For the long-range electrostatic interactions, the particle-mesh Ewald (PME) method was used (Darden et al., 1993). The linear constraint solver (LINCS) algorithm was used to preserve covalent bond lengths (Hess et al., 1997). Prior to all MD simulations, the steepest-descent algorithm was used to minimize the energy of the initial configurations.

\section{Results}

\subsection{MD simulations}

We first considered the location (depth) and orientation of Px molecules inside membranes composed of pure POPC or containing an admixture of Chol. Figures S2 and S3 (Supplementary Material) show trajectories of the mass center of Px molecules along the membrane normal ( $z$ coordinate). A limited number of simulated Px molecules does not allow us to draw quantitative information from the figures. However, several qualitative conclusions regarding the entry of Px into the bilayer can be drawn. Both neutral tautomers PxA and PxE had a low tendency to move from the aqueous phase to the POPC bilayer (systems M1); from 13 molecules initially located in the aqueous phase only 3 PxA migrated into the POPC membrane, while this was not seen to ever occur for the PxE tautomer. The molecules in cationic and zwitterionic forms showed much more dynamic behavior: translocations in both directions, from water to the membrane and from the membrane into the water, were observed. The incorporation of Chol into the POPC bilayer 
Wilkosz et al. European Journal of Pharmaceutical Sciences 2017, 100, 116-125. http://dx.doi.org/10.1016/j.ejps.2017.01.007

(systems M2) was seen to facilitate the entrance of both neutral tautomers into the membrane; from 26 molecules initially placed in water 7 of them (5 PxA and 2 PxE) translocated into the POPC/Chol membrane. On the contrary, for the Px forms bearing charges no successful entrance into the bilayer was observed. The trajectories for the PxA and PxE molecules remaining in the aqueous phase showed colocalization, indicating the formation of dimers or oligomers. The structures of Px clusters that formed in the bulk water phase are shown in Figure 2A-C. The formation of aggregates slowed down the translocation of the molecules from water to the membrane to a time scale much longer than the current simulations. Both PxA and PxE molecules, initially inserted in the center of the POPC and POPC/Chol bilayers (systems M1b and M2b), were seen to migrate immediately to the interface between polar and hydrophobic membrane regions and remained there for the rest of the simulation time. In a similar manner, the molecules that did enter the bilayer from the aqueous phase diffused to the same interface. For PxZw and $\mathrm{Px}^{+}$, translocations from the membrane to the water phase were observed.

For the case of system M3 (Figure S4), three events of translocation of neutral tautomers from water to the membrane were observed and also molecules placed in the bilayer center moved toward the headgroup region. For the cationic form, one event of translocation from bilayer to the water phase was observed.

\subsubsection{Px Location in Membranes}

Selected snapshots taken at the end of the simulations, illustrating the location and orientation of the drug in the lipid bilayers, are shown in Figures 1B, 1D, and 1F. They indicate that the preferred position and arrangement of Px were dependent on the type of bilayers. To elucidate the position of the drug with respect to the lipid molecules, we calculated their mass density profiles across the membrane. 
Wilkosz et al. European Journal of Pharmaceutical Sciences 2017, 100, 116-125. http://dx.doi.org/10.1016/j.ejps.2017.01.007

Figure 3 shows the density profiles along the membrane normal for the various Px forms and selected atoms of POPC (phosphors, nitrogens, and carbonyl oxygens). In system M1 (the POPC bilayer) the profiles of carbonyl, phosphate and choline groups had the maxima at 1.52, 1.95, and $2.23 \mathrm{~nm}$ from the bilayer center, respectively. The PxA and PxE molecules that were inside the membrane were located preferentially below the carbonyl groups, at the distance of $1.13 \mathrm{~nm}$ from the bilayer center. The $\mathrm{PxZw}$ and $\mathrm{Px}^{+}$forms were located at a shallower position in the bilayer at the distance of 1.42 and $1.38 \mathrm{~nm}$, respectively. As expected, the presence of Chol in the POPC membrane had some influence on the bilayer thickness, as a result the profiles of carbonyl, phosphate and choline groups had the maxima at 1.80, 2.22, and $2.56 \mathrm{~nm}$ from the bilayer center, respectively. In system M2 (the POPC/Chol bilayer) neutral Px molecules were also located below the lipid carbonyl groups, but the favorite position of tautomer PxA was slightly deeper in the bilayer $(1.21 \mathrm{~nm})$ in comparison to that for the PxE tautomer $(1.42 \mathrm{~nm})$. For the case of PxZw and $\mathrm{Px}^{-}$, the molecules were located in the polar region close to or above the carbonyl groups. In both systems M1 and M2, we observed that the density profiles of PxA and PxE also had maxima in the aqueous phase close to the lipid headgroup indicating that these tautomers interact with the lipid polar groups. However, the densities do not fall to zero values in the aqueous phase indicating that the neutral forms of Px remain in water, probably as aggregates. This is in agreement with the trajectories of the center of mass shown in Figure S2 and S3.

Figure $3 \mathrm{C}$ shows the average mass density profiles for carbonyl, phosphate, and choline groups of the lipids, the PEG chains, and the different forms of Px in the POPC/DSPE-PEG bilayer (system M3). For all forms of Px, two maxima can be observed in the density profiles: one at a distance of 1.07-1.61 nm corresponding to Px in the bilayer and another at a distance of 3.34- 
Wilkosz et al. European Journal of Pharmaceutical Sciences 2017, 100, 116-125. http://dx.doi.org/10.1016/j.ejps.2017.01.007

$4.04 \mathrm{~nm}$ from the bilayer center, corresponding to Px in the PEG layer. Thus, the drug molecules preferentially located below carbonyl groups similar to system M1 and M2 as indicated by the first maximum. The second maximum completely overlaps with the profiles of PEG, showing that the drug molecules that did not enter the lipid bilayer were in the PEG corona, as it is illustrated in Figure 1F.

\subsubsection{Orientation of $P x$ in Membranes}

To describe the orientation of Px molecules inside the membranes, we used two parameters: the angle $\theta_{\text {long }}$ between the Px long axis (the long vector, see Figure 4) and the bilayer normal, and the angle $\theta_{\text {short }}$ between the short vector (between the $\mathrm{S}$ atom and the enolic $\mathrm{C}$ atom) and the bilayer normal. The normalized distributions of the angles for Px molecules, which entered the membrane in different systems are shown in Figure 4. Several conclusions regarding the orientation can be drawn from these figures: (i) generally, all presented distributions are rather broad showing that Px has great orientational freedom in all bilayers; (ii) the orientation is dependent on the form of the drug molecule, (iii) the molecules adopt orientations, in which hydrophilic groups are exposed to the water.

\subsection{4. $P x-P x$ Interactions}

As mentioned earlier in this article, Px forms the aggregates in the bulk water phase.

Unfortunately, our statistics regarding this phenomenon are limited, thus we are not able to accurately calculate the average lifetime of the clusters, however, during the simulations we observed several events of both the formation and decay of clusters. The observed clusters were formed due to $\pi$-stacking interactions, but the exact geometrical arrangements varied from case to case (see Figure 2 and Figure S5 for examples). In one case, we observed a cluster formed by 
Wilkosz et al. European Journal of Pharmaceutical Sciences 2017, 100, 116-125. http://dx.doi.org/10.1016/j.ejps.2017.01.007

three molecules of PxA (see the trajectory of the center of mass shown in Figure S2A) entering the membrane core as a single unit, that remained intact until the end of the simulation run (the structure shown in Figure 2D).

\subsection{Experimental measurements}

\subsubsection{Px entrapment in POPC membrane - LSCM Observations.}

POPC and POPC/DSPE-PEG GUVs were treated with Px for two hours at the drug/lipid ratio of 0.005 . The partitioning of Px into the liposomal bilayer was directly confirmed through visualization using the LSCM microscopy technique. As an example, Figure 5 shows the DIC contrast and fluorescence confocal micrographs of the POPC/DSPE-PEG liposomes treated with Px. The liposomes exhibited fluorescence characteristics of the drug upon excitation at $405 \mathrm{~nm}$. This can be seen as clear evidence that Px can penetrate into the lipid bilayer or PEG corona.

\subsubsection{Determination of liposome binding constant}

The partitioning of the drug molecules between the lipid vesicles and the aqueous phase can be quantitatively described by the so-called binding constant $\left(K_{\mathrm{b}}\right)$ (Nawalany et al., 2008).

Considering the fact that in the partitioning process the partly aggregated Px can participate, we determined the apparent binding constant, defined as

$$
K_{\mathrm{b}}=\frac{c_{\mathrm{L}}}{c_{\mathrm{w}}[\mathrm{L}]}
$$

where $c_{\mathrm{L}}$ and $c_{\mathrm{w}}$ are Px concentrations in the lipid vesicles and in the aqueous phase, respectively, and $[\mathrm{L}]$ is the concentration of the lipid.

Two series of aqueous solutions containing Px at the constant concentration and POPC or POPC/DSPE-PEG at the concentrations [L] varying from 0 to ca. $0.35 \mathrm{mg} / \mathrm{mL}$ were prepared and 
Wilkosz et al. European Journal of Pharmaceutical Sciences 2017, 100, 116-125. http://dx.doi.org/10.1016/j.ejps.2017.01.007

the steady-state emission spectra were measured. Figure 6 presents typical sets of spectra obtained for Px in the presence of lipid at various concentrations. For the POPC liposomes, pronounced spectral changes were observed when lipid vesicles were added to the Px solution. The shape of the fluorescence spectra became similar to that characteristic of the drug in organic solvents such as DMF (Figure S6) and this change was accompanied by an amplification of the fluorescence intensity. We considered the partitioning of the Px molecules into a lipid environment, whose polarity is similar to that of DMF, as the cause of the changes we observed in the fluorescence spectra. Therefore, determination of the binding constant was based on the observed spectral changes. Figure 6A shows the fluorescence intensities at $445 \mathrm{~nm}$ versus [L] and the line fitted to equations 1 (see the section 2.4). The calculated liposome-binding constant was equal to $K_{\mathrm{b}}=33.2 \pm 2.9(\mathrm{mg} / \mathrm{mL})^{-1}$. This means that at a lipid concentration of $0.03 \mathrm{mg} / \mathrm{mL}$ half of the Px molecules are located in the lipid phase and the other half in the aqueous phase; at the POPC concentration of $2.5 \mathrm{mg} / \mathrm{mL}$ up to $98.8 \%$ of the drug is enclosed in the lipid bilayer.

For the POPC/DSPE-PEG system (Figure 6B), the amplification of fluorescence spectra upon the addition of lipid vesicles was less pronounced and their shapes were similar to that observed in more polar organic solvents such as, for example, in ethanol (Yoon et al., 1988). The changes in the fluorescence spectra undoubtedly resulted from the transfer of Px molecules from the aqueous phase to the PEG corona of the PEGylated liposomes. In such a way, the aggregation of the drug molecules was reduced and the fluorescence intensity increased. The fluorescence intensity at $445 \mathrm{~nm}$ as a function of $[\mathrm{L}]$ is shown in the inset of Figure $6 \mathrm{~B}$ together with the line that was fitted to this data using equation 1 . The calculated value of $K_{\mathrm{b}}$ was $0.55 \pm 0.3$ $(\mathrm{mg} / \mathrm{mL})^{-1}$

\section{Discussion}


Wilkosz et al. European Journal of Pharmaceutical Sciences 2017, 100, 116-125. http://dx.doi.org/10.1016/j.ejps.2017.01.007

In this study, we considered the behavior of an ionizable drug at the surface of a zwitterionic membrane, which was used as a model system for protein-free cell membranes. Since PEGylation and the incorporation of Chol can be used to stabilize liposomes, we also clarified the effect of the presence of a PEGylated lipid and Chol on the location and orientation of the drug molecules in the membrane. The PEG length was chosen to be 45 units, corresponding to a molecular weight of about $2000 \mathrm{Da}$, since PEG of this molecular weight is the most commonly used in PEGylated liposomes for pharmaceutical applications (Maruyama et al., 1991).

Piroxicam has dynamic structural features. It can exist in seven different neutral tautomeric forms and the tautomeric shifts result from fast internal proton transfers, being unusually sensitive to changes in solvent (polarity) and temperature (Ivanova et al., 2015). Using the quantum-chemical calculations and UV-vis measurements it was shown that the enol-amide tautomer PxA (Scheme 1) is the most stable form in organic solvents (ethanol and DMSO) and it is stabilized as a sandwich-type dimer. Upon addition of water the dimer is partially destroyed and the tautomeric equilibrium is shifted to the zwitterionic form. Moreover, Px has in its structure an imine nitrogen atom in the pyridine ring that is able to attach a proton, forming the cationic form, and an enolic $\mathrm{OH}$ group that can dissociate, thus forming an anionic form in the aqueous solution. A simplified diagram of the acid-basic equilibria of Px in the aqueous environments is shown in Scheme 1. The $\mathrm{p} K_{\mathrm{a}} \mathrm{s}$ of Px have been determined previously. Using UV-spectrophotometric titrations, Bernhard and Zimmermann determined two $\mathrm{p} K_{\mathrm{a}}$ values of 1.86 and 5.46 (Bernhard and Zimmermann, 1984). Shalaeva et al. reported slightly different values of 1.81 and 5.28 determined using a multiplexed capillary electrophoresis (Shalaeva, et al., 2008). Furthermore, the values of $2.12 \pm 0.04$ and $5.31 \pm 0.03$ were reported by RodriguezBarrientos (Rodríguez-Barrientos et al., 2009). On the basis of the reported $\mathrm{p} K_{\mathrm{a}}$ values we 
Wilkosz et al. European Journal of Pharmaceutical Sciences 2017, 100, 116-125. http://dx.doi.org/10.1016/j.ejps.2017.01.007

calculated the molar fractions of the different acid-base forms as a function of pH. Figure S7 shows that at $\mathrm{pH}$ about 4.0, $\mathrm{Px}$ is present mainly in the neutral/zwitterionic form. Therefore, our measurements were carried out at this $\mathrm{pH}$ value.

Our experimental results proved that the neutral/zwitterionic form of Px can penetrate into the zwitterionic membrane. However, the kinetics of this process is rather slow, because the incubation time needed to reach equilibrium was long (a few hours). The high affinity of the Px neutral/zwitterionic form to zwitterionic membranes was previously demonstrated using 1,2dimyristoyl-sn-glycero-3-phosphocholine (DMPC) liposomes (Chakraborty et al., 2005). It was shown that the partition coefficient of the Px neutral form to the DMPC membrane is 8.5 times higher than that of its anionic form. Our simulation results are in line with experimental findings. As we have shown, the PxA, PxE, and PxZw molecules initially placed in the lipid bilayer (systems M1b and M2b) did not tend to move into the aqueous phase, which indicates the high affinity of these Px forms for the zwitterionic lipid membrane. On the other hand, the translocation of the molecules from water to the membrane was slowed down by the formation of aggregates in the aqueous phase. This indicates that mainly monomeric molecules are capable to partition to the membrane.

The arrangement and depth-localization of Px inside a lipid bilayer has been poorly recognized in the literature. We have found only one study on the depth-localization of Px in the bilayer. The interaction of Px with 1,2-dipalmitoyl-sn-glycero-3-phosphocholine (DPPC) liposomes at pH 7.4 and 5.0 was studied using fluorescence steady-state anisotropy (Nunes et al., 2011). The results suggest that the anionic form of $\mathrm{Px}(\mathrm{pH} 7.4)$ is located in the more highly ordered region of the acyl chains near the headgroups (C1-C9). Also, at pH 5.0, the Px molecules locate in the chain region closer to the headgroups (C2-C8), but they penetrate deeper than at $\mathrm{pH}$ 7.4. MD 
Wilkosz et al. European Journal of Pharmaceutical Sciences 2017, 100, 116-125. http://dx.doi.org/10.1016/j.ejps.2017.01.007

simulations allowed us to determine the preferred location of the Px forms in the membrane. Both Px tautomers were localized at the same depth in the POPC bilayer, about $0.4 \mathrm{~nm}$ below the carbonyl groups of the acyl chains. As expected PxZw is located in a more shallow fashion in the POPC membrane and its position coincides with that of the carbonyl groups of the lipid. Thus, the neutral and zwitterionic forms of Px preferentially localized in the membrane close to the headgroup region, which is consistent with the earlier experimental findings (Nunes et al., 2011). The long axis of PxA, PxE, and PxZw molecules had great orientational freedom with a tendency to align parallel to the membrane surface (the maxima of $\theta_{\text {long }}$ are in the range of 70$110^{\circ}$, Figure 4), as shown in Figure 1B for tautomer PxA. At first sight such location and orientation might look unreasonable as this part of the bilayer is the most ordered and rigid. However, the parallel arrangement of Px in the POPC bilayers can be explained by the drug structure and its interactions with water and with the polar groups of the lipid. Px contains several polar groups that are regularly distributed along the molecular skeleton. These groups can form hydrogen bonds (H-bonds) with water and with the polar headgroups of a membrane. Therefore, the orientation of Px within the membrane should be analyzed in terms of the formation of H-bonds with water and lipid molecules. The averaged numbers of $\mathrm{H}$-bonds are given in Table S1. As all polar groups of POPC are hydrogen acceptors, the formation of Hbonds in the Px-POPC pair is limited only to hydrogen donors of Px. For example, for the PxAPOPC pair, the H-bonds are created between nonester oxygen in the phosphate group of POPC and the amide $\mathrm{NH}$ group $(\mathrm{Px}-\mathrm{NH} \cdots \mathrm{POPC})$ or the hydroxyl group $(\mathrm{Px}-\mathrm{OH} \cdots \mathrm{POPC})$ of the drug. In the case of $\mathrm{Px}$-water pairs, $\mathrm{H}$-bonds can be formed between all polar groups of $\mathrm{Px}$ and water molecules since water is both hydrogen donor and acceptor. On average, the Px molecules are partially hydrated by 1.1-3.6 water molecules. Thus, the adopted orientation is optimal as it 
Wilkosz et al. European Journal of Pharmaceutical Sciences 2017, 100, 116-125. http://dx.doi.org/10.1016/j.ejps.2017.01.007

reduces the degree of contact between water and the hydrophobic part of the Px molecule and is additionally stabilized by H-bonds of the lipid headgroups and polar groups of Px.

The incorporation of Chol had little effect on the depth of Px location in the membrane. The shape of the density profile of Px indicates also one preferential location within the POPC/Chol membrane. However, PxA was located slightly deeper within the membrane compared to PxE. On the contrary, the presence of Chol in the bilayer significantly affected the orientation of the drug molecules. The PxA, PxE, and PxZw molecules adopted an orientation where their long axis was parallel to the lipid molecules (the maxima of $\theta_{\text {long }}$ are at the angles $<30^{\circ}$ or $>150^{\circ}$, Figure 4) or almost parallel to the membrane surface. The differences in the arrangements of the Px molecules in the two bilayers can be explained by considering the well-known ordering effect of Chol on the bilayer structure (Róg et al., 2014). The presence of Chol increases the order of the acyl tails and both decreases the lipid mobility and increases the rigidity of the lipid bilayers (see the results for a POPC bilayer obtained with the same setup) (Róg et al., 2014). The average tilt of the cholesterol molecules in our studies was $\sim 14.6^{\circ}$ and since it is a rigid molecule its orientation was restricted and did not fluctuate significantly.

The earlier fluorescence spectroscopy studies performed on living splenocyte cells indicated that Px increased the membrane fluidity in a concentration dependent manner (Ferreira et al., 2005). MD simulation study by Markiewicz et al. demonstrated a small increase in the surface area per lipid, but at the same time a small increase in the order parameter of hydrocarbon tails was observed (Markiewicz et al., 2011). As both parameters are indirectly related to fluidity, a comparison between the experimental and MD studies is not straightforward. In our simulations, the differences between the properties of pure POPC bilayer and that containing the incorporated drug were so small that they are below the precision of our calculations. This is due to very low 
Wilkosz et al. European Journal of Pharmaceutical Sciences 2017, 100, 116-125. http://dx.doi.org/10.1016/j.ejps.2017.01.007

concentration of Px in the considered by us bilayers (i.e. 4 times lower than in MD simulations described in Markiewicz et al., 2011).

An interesting result found from the simulations is that Px can form aggregates in both bulk water and in the lipid bilayer. It is well known that Px is poorly soluble in water. Also, the aggregation phenomenon of PxA in polar organic solvents has been previously studied, and the dimerization equilibrium constant, $K_{\mathrm{D}}$, was found to be $7.2 \times 10^{5}$ and $3.2 \times 10^{4} \mathrm{M}^{-1}$ in ethanol and DMSO, respectively (Ivanova et al., 2015). The distance between Px molecules in the dimer was determined experimentally to be 0.34 and $0.32 \mathrm{~nm}$ in ethanol and in DMSO, respectively. Thus, the tendency for dimerization is very strong and the molecules in organic solutions are present mostly in the aggregated form. These results strongly support the existence of Px aggregate in the lipid membrane, that was observed during the simulation and is illustrated in Figure 2D. The distance of the Px rings in these clusters was found to vary between 0.3 and 0.4 $\mathrm{nm}$.

POPC is the key phospholipid of gastric mucus and mucosa, and the POPC/Chol bilayer is a relevant model of the phospholipid oligolamellar structures of the gastric mucosa hydrophobic barrier as well as the membrane of gastric mucosa cells (Markiewicz et al., 2011). Under highly acidic conditions, for example inside the stomach ( $\mathrm{pH} 1-2)$, about 50-80 \% of Px molecules occur in the cationic form (Figure S7). Our MD simulations revealed that $\mathrm{Px}^{+}$had a lower affinity for the bilayer compared to the neutral forms, because three of the six cations initially placed in the bilayer escaped to the aqueous phase during the simulation period (Figure S2 and S3). However, the appearance of the positive charge on the pyridine ring did not prevent the insertion of Px into the lipid environment of zwitterionic liposomes. We observed that four of the eighteen cations initially placed in the aqueous phase managed to enter the membrane and stayed 
Wilkosz et al. European Journal of Pharmaceutical Sciences 2017, 100, 116-125. http://dx.doi.org/10.1016/j.ejps.2017.01.007

in this environment for the remaining time of simulation. The behavior of $\mathrm{Px}^{+}$can be explained by considering its interaction with both water and the lipid headgroups. While the Px cations are hydrated by the number of water molecules close to that of the neutral forms, the number of $\mathrm{H}$ bonds formed between $\mathrm{Px}^{+}$and POPC is considerably higher in comparison to the neutral forms of Px. This indicates that Px in its cationic form can interact with phospholipid bilayers causing alterations in their structural properties under acidic conditions, especially reducing their hydrophobicity as the $\mathrm{Px}^{+}$molecules incorporated to the $\mathrm{POPC} / \mathrm{Chol}$ bilayer are hydrated by $\sim 2$ water molecules. Lower hydrophobicity of gastric mucosa can increase its permeability and result in its lowered resistance to luminal acid (Lichtenberger, 2001).

The $K_{\mathrm{b}}$ values indicate that Px in the neutral forms has a lower affinity for partitioning into the hydrophobic core of the PEGylated membrane in comparison to the conventional liposomes. Thus, the presence of the PEG corona at the surface of liposomes hinders entering of Px into the lipid membrane. However, the intensity of the fluorescence spectrum of the drug increased with the increasing concentration of the liposomes, showing that Px interacted with the POPC/DSPEPEG liposomes. Moreover, the direct microscopic observations showed that the drug was accumulated at the membrane (Figure 5), thus confirming that Px can interact with PEGylated bilayers. These experimental findings can be explained assuming that with the increasing content of the POPC/DSPE-PEG liposomes the Px molecules translocated mainly to the PEG corona, thus the aggregation was reduced.

The results of the simulations confirm the embedding of the drug molecules inside the PEG layer. The mass density profile of PEG chains (Figure 3C) has two maxima: one outside of a bilayer and another inside a lipid membrane. This indicates that a portion of the PEG chains penetrated the lipid core of the membrane being in the liquid state, which is in agreement with 
Wilkosz et al. European Journal of Pharmaceutical Sciences 2017, 100, 116-125. http://dx.doi.org/10.1016/j.ejps.2017.01.007

our previous simulations (Dzieciuch et al., 2015; Stepniewski et al., 2011). The incorporation of PEGylated lipids into the membrane had a significant impact on Px location. The Px molecules have two preferred positions: inside the membrane below the carbonyl groups and in the PEG layer. The location of Px in the PEG corona is stabilized by hydrogen bonds between oxygens of PEG and the hydroxyl ((PEG)O $\cdots \mathrm{HO}-\mathrm{Px})$ or amino ((PEG)O $\cdots \mathrm{HN}-\mathrm{Px})$ groups of Px, especially in the case of PxZw and $\mathrm{Px}^{+}$(Table S1). Additionally, the hydrophobic ethylene groups of PEG chain interacted with the nonpolar surface of the molecule. As a result, Px molecules were wrapped with the PEG chains.

\section{Conclusions}

Px is a frequently used non-steroidal anti-inflammatory drug characterized by low solubility, which limits its adsorption from intestines. Px has numerous side effects including e.g. bleeding from the stomach and intestines after oral administration. As with many drugs where toxicity is a serious issue, the use of liposome based delivery systems has been considered as a way to alleviate this problem (Bunker et al., 2016). In this study, we used comprehensive MD simulations and fluorescence experiments to investigate the interaction of Px, a model for ionizable water-insoluble drugs, with various lipid bilayers. We considered three lipid membranes: zwitterionic (composed of POPC), zwitterionic doped with Chol (composed of POPC and Chol), and PEGylated (composed of POPC and DSPE-PEG).

Our experiments have shown that the neutral/zwitterionic form of Px can penetrate into zwitterionic and PEGylated membranes. Direct microscopic observations confirmed the partitioning of the drug into the liposomal bilayers. However, studies by fluorescence spectroscopy, revealed considerable differences in the behavior of Px embedded in these two types of membranes. We observed that in the PEGylated membrane Px were exposed to the more 
Wilkosz et al. European Journal of Pharmaceutical Sciences 2017, 100, 116-125. http://dx.doi.org/10.1016/j.ejps.2017.01.007

polar environment in comparison to the conventional liposomes, indicating that the drug molecules located mainly to the PEG corona.

Our MD simulations provide an insight into the nature of Px - lipid membrane interactions at the molecular level. In the MD simulations, we considered two low energy uncharged tautomers: PxA and PxE. In aqueous solutions Px adopts charged forms under certain conditions, for example zwitterionic and cationic forms dominate in the acidic environment inside the stomach. We thus additionally considered these two forms. In agreement with experiments, we found that Px can enter lipid bilayers. Uncharged forms of PxA and PxE were observed to locate below the carbonyl groups of the lipids, while the charged forms located to the level of the carbonyl groups, thus closer to the water phase. Preferential location of drugs including other NSAIDs to the water-membrane interface was observed in both experimental and MD simulation studies (Cramariuc et al., 2012). Cholesterol was found to have no influence on the Px location. For the PEGylated bilayers, our MD simulations revealed that Px molecules locate to the lipid headgroup region of the membrane and within the PEG layer. Px molecules are wrapped by PEG chains attached to lipid molecules. MD simulations indicate that Px has great orientational freedom in the lipid bilayers, however the presence of cholesterol decreases this orientational freedom. The dominant orientation of $\mathrm{Px}$ is parallel to the bilayer surface, thus allowing for all polar Px groups to interact with both the polar lipid groups and water by hydrogen bonding.

Concluding, both MD simulations and experimental methods showed that Px molecules preferentially locate in both conventional and PEGylated lipid membranes. Moreover, the PEG corona around lipid vesicles can serve as an additional volume for accumulation of drug molecules in sterically stabilized liposomes thus increasing the efficiency of loading. 
Wilkosz et al. European Journal of Pharmaceutical Sciences 2017, 100, 116-125. http://dx.doi.org/10.1016/j.ejps.2017.01.007

\section{AUTHOR INFORMATION}

The authors declare no competing financial interest.

\section{Author Contributions}

All authors contributed to design of the studies and the manuscript preparation. NW and MC performed experiments. SR performed all MD simulations and analyzed MD trajectories. MK and TR supervised all studies. All authors have given approval to the final version of the manuscript.

\section{ACKNOWLEDGMENTS}

This project was supported by the National Science Centre Poland on the basis of the decision number DEC-2012/07/B/ST5/00913. The research was carried out with the equipment purchased thanks to the financial support of the European Regional Development Fund in the framework of the Polish Innovation Economy Operational Program (contract No. POIG.02.01.00-12-023/08). CSC - Finnish IT Centre for Scientific Computing (Espoo, Finland) is acknowledged for computer resources. European Research Council (Advanced Grant project CROWDED-PROLIPIDS) and the Academy of Finland Center of Excellence program are thanked for financial support. 
Wilkosz et al. European Journal of Pharmaceutical Sciences 2017, 100, 116-125. http://dx.doi.org/10.1016/j.ejps.2017.01.007

\section{Figure Captions}

Scheme 1. Chemical structure of two tautomers of the Px neutral form and acid-base equilibria of the different forms of Px. The values of $\mathrm{p} K_{1}$ and $\mathrm{p} K_{2}$ are equal to 1.86 and 5.46, respectively (Tsai et al., 1993).

Figure 1. Snapshots of the systems consisting of PxA molecules in different membranes: in POPC (system M1b) at the beginning (A) and after $300 \mathrm{~ns}$ of simulation (B), in POPC/Chol (4:1) (system M2b) at the beginning (C) and after $300 \mathrm{~ns}$ of simulation(D), and in POPC/DSPE-PEG (system M3) at the beginning (E) and after 500 ns of simulation (F). PxA molecules are shown in red, the POPC molecules are shown as transparent-gray, with dark grey spheres for the phosphate groups and the Chol molecules and PEG chains are shown in yellow and light gray, respectively. For clarity, water and ions are not shown.

Figure 2. Snapshots showing Px clusters that formed during the simulations. The clusters of PxA molecules formed in bulk water in system M1b at $190 \mathrm{~ns}$ (A), at $260 \mathrm{~ns}$ (B), at $300 \mathrm{~ns}$ (C). The cluster of PxA in the POPC/Chol bilayer in system M1b at 300 ns (D).

Figure 3. Mass density profiles of Px and the selected lipid atoms along the bilayer normal as averaged over the last $200 \mathrm{~ns}$ of the trajectories and also over the simulated system: M1 (A), M2 (B), and M3 (C).

Figure 4. Probability distribution of the angles $\theta_{\text {long }}(\mathrm{A}, \mathrm{C}$, and $\mathrm{E})$ and $\theta_{\text {short }}(\mathrm{B}, \mathrm{D}$, and $\mathrm{F})$ in system M1 (A, B), M2 (C, D) and M3 (E, F) for PxA (black lines), PxE (red lines), Px ${ }^{+}$(blue lines), and PxZw (green lines). Definitions of long and short vectors are shown on the chemical structure of PxA. Color shades represent the standard deviations calculated from independent runs. 
Wilkosz et al. European Journal of Pharmaceutical Sciences 2017, 100, 116-125. http://dx.doi.org/10.1016/j.ejps.2017.01.007

Figure 5. Differential interference contrast (DIC) (A) and LSCM (B) micrographs of the POPC/DSPE-PEG liposomes $\left(c_{\text {lipid }}=2.5 \mathrm{mg} / \mathrm{mL}\right)$ treated with $\mathrm{Px}\left(c_{\mathrm{Px}}=16.5 \mu \mathrm{M}\right)$. The scale bar corresponds to $5 \mu \mathrm{m}$.

Figure 6. Fluorescence spectra of $\mathrm{Px}\left(c_{\mathrm{Px}}=32.9 \mu \mathrm{M}, \lambda_{\mathrm{ex}}=377 \mathrm{~nm}\right)$ in buffer $(\mathrm{pH} 4.0$, dotted lines) and in the presence of increasing concentrations of POPC (A) or POPC/DSPE-PEG (B) liposomes (solid lines). The inserts show the intensity of fluorescence maximum at $445 \mathrm{~nm}$ as a function of the added lipid concentration, as well as the line fitted to equation 1.

\section{References}

van Balen, G.P., Martinet, C.M., Caron, G., Bouchard, G., Reist, M., Carrupt, P.-A., Fruttero, R., Gasco, A., Testa, B., 2004. Liposome/Water Lipophilicity: Methods, Information Content, and Pharmaceutical Applications. Med. Res. Rev. 24, 299-324.

Banerjee, R., Sarkar, M., 2002. Spectroscopic studies of microenvironment dictated structural forms of piroxicam and meloxicam. J. Luminescence 99, 255-263.

Bernhard, E., Zimmermann, F., 1984. Contribution to the understanding of oxicam ionization constants. Arzneimittel-Forschung 34, 647-648.

Bunker, A., Magarkar, A., Viitala, T., 2016. Rational design of liposomal drug delivery systems, a review: Combined experimental and computational studies of lipid membranes, liposomes and their PEGylation. Biochim. Biophys. Acta - Biomembranes 1858, 2334-2352. 
Wilkosz et al. European Journal of Pharmaceutical Sciences 2017, 100, 116-125. http://dx.doi.org/10.1016/j.ejps.2017.01.007

Canto, G.S., Dalmora, S.L., Oliveira, A.G., 1999. Piroxicam Encapsulated in Liposomes: Characterization and In Vivo Evaluation of Topical Anti-inflammatory Effect. Drug Dev. Ind. Pharm. 25, 1235-1239.

Chakraborty, H., Roy, S., Sarkar, M., 2005. Interaction of oxicam NSAIDs with DMPC vesicles: differential partitioning of drugs. Chem. Phys. Lipids 138, 20-28.

Chiong, H.S., Yong, Y.K., Ahmad, Z., Sulaiman, M.R., Zakaria, Z.A., Yuen, K.H., Hakim, M.N., 2013. Cytoprotective and enhanced anti-inflammatory activities of liposomal piroxicam formulation in lipopolysaccharide-stimulated RAW 264.7 macrophages. Int. J. Nanomed. 8, $1245-1255$.

Cramariuc, O., Róg, T., Vattulainen, I., 2012. Drug-lipid membrane interaction mechanisms revealed through molecular simulations. Curr. Phys. Chem. 2, 379-400.

Darden, T., York, D., Pedersen, L., 1993. Particle mesh Ewald: An $\underline{N} \cdot \log (N)$ method for Ewald sums in large systems. J. Chem. Phys. 98, 10089-10092.

Dzieciuch, M., Rissanen, S., Szydłowska, N., Bunker, A., Kumorek, M., Jamróz, D., Vattulainen, I., Nowakowska, M., Róg, T., Kepczynski, M., 2015. PEGylated Liposomes as Carriers of Hydrophobic Porphyrins. J. Phys. Chem. B 119, 6646-6657.

Ferreira, H., Lúcio, M., Lima, J.L.F.C., Cordeiro-da-Silva, A., Tavares, J., Reis, S., 2005. Effect of anti-inflammatory drugs on splenocyte membrane fluidity. Anal. Biochem. 339, 144149. 
Wilkosz et al. European Journal of Pharmaceutical Sciences 2017, 100, 116-125. http://dx.doi.org/10.1016/j.ejps.2017.01.007

Ferreira de Souza, K., Martins, J.A., Pessine, F.B.T., Custodio, R., 2010. A theoretical and spectroscopic study of conformational structures of piroxicam. Spectrochim. Acta A 75, 901-907.

Hess, B., Bekker, H., Berendsen, H.J.C., Fraaije, J.G.E.M., 1997. LINCS: A linear constraint solver for molecular simulations. J. Comput. Chem. 18, 1463-1472.

Hess, B., Kutzner, C., van der Spoel, D., Lindahl, E., 2008. GROMACS 4: Algorithms for highly efficient, load-balanced, and scalable molecular simulation. J. Chem. Theory Comput. 4, $435-447$.

Hoover, W.G., 1985. Canonical Dynamics: Equilibrium Phase-Space Distributions. Phys. Rev. A $31,1695-1697$.

Ivanova, D., Deneva, V., Nedeltcheva, D., Kamounah, F.S., Gergov, G., Hansen, P.E., Kawauchi, S., Antonov, L., 2015. Tautomeric transformations of piroxicam in solution: a combined experimental and theoretical study. RSC Adv. 5, 31852-31860.

Jolliet, P., Simon, N., Brée, F., Urien, S., Pagliara, A., Carrupt, P.A., Testa, B., Tillement, J.P., 1997. Blood-to-Brain Transfer of Various Oxicams: Effects of Plasma Binding on Their Brain Delivery. Pharm. Res. 14, 650-656.

Jorgensen, W.L., Chandrasekhar, J., Madura, J.D., Impey, R.W., Klein, M.L., 1983. Comparison of simple potential functions for simulating liquid water. J. Chem. Phys. 79, 926935. 
Wilkosz et al. European Journal of Pharmaceutical Sciences 2017, 100, 116-125. http://dx.doi.org/10.1016/j.ejps.2017.01.007

Jorgensen, W.L., Maxwell, D.S., Tirado-Rives, J., 1996. Development and Testing of the OPLS All-Atom Force Field on Conformational Energetics and Properties of Organic Liquids. J. Am. Chem. Soc. 118, 11225-11236.

Kepczynski, M., Nawalany, K., Kumorek, M., Kobierska, A., Jachimska, B., Nowakowska, M., 2008. Which Physical and Structural Factors of Liposome Carriers Control Their DrugLoading Efficiency? Chem. Phys. Lipids 155, 7-15. ZAMIANA KOLEJNOŚCI

Kępczyński, M., Pandian, R.P., Smith K.M., Ehrenberg, B., 2002. Do Liposome-binding Constants of Porphyrins Correlate with Their Measured and Predicted Partitioning Between Octanol and Water? Photochem. Photobiol. 76, 127-134.

Kulig, W., Pasenkiewicz-Gierula, M., Róg, T., 2015. Topologies, structures and parameter files for lipid simulations in GROMACS with the OPLS-aa force field: DPPC, POPC, DOPC, PEPC, and cholesterol. Data in Brief 5, 333-336.

Kulig, W., Pasenkiewicz-Gierula, M., Róg. T., 2016. Cis and trans unsaturated phosphatidylcholine bilayers: A molecular dynamics simulation study. Chem. Phys. Lipids 195, $12-20$.

Lasic, D.D., 1998. Novel Applications of Liposomes. Trends Biotechnol. 16, 307-321.

Lee, E.H., Hsin, J., Sotomayor, M., Comellas, G., Schulten, K., 2009. Discovery through the computational microscope. Structure 17, 1295-1306.

Li, Y.-C., Rissanen, S., Stepniewski, M., Cramariuc, O., Róg, T., Mirza, S., Xhaard, H., Wytrwal, M., Kepczynski, M., Bunker, A., 2012. Study of interaction between PEG carrier and 
Wilkosz et al. European Journal of Pharmaceutical Sciences 2017, 100, 116-125. http://dx.doi.org/10.1016/j.ejps.2017.01.007

three relevant drug molecules: piroxicam, paclitaxel, and hematoporphyrin. J. Phys. Chem. B. $116,7334-7341$.

Lichtenberger, L. M., 2001. Where is the evidence that cyclooxygenase inhibition is the primary cause of nonsteroidal anti-inflammatory drug (NSAID)-induced gastrointestinal injury?: Topical injury revisited. Biochem. Pharmacol. 61, 631-637.

Maciejewski, A., Pasenkiewicz-Gierula, M., Cramariuc, O., Vattulainen, I., Róg, T., 2014. Refined OPLS-All-Atom Force Field for Saturated Phosphatidylcholine Bilayers at Full Hydration. J. Phys. Chem. B. 118, 4571-4581.

Markiewicz, M., Pasenkiewicz-Gierula, M. 2011. Comparative model studies of gastric toxicity of nonsteroidal anti-inflammatory drugs. Langmuir 27, 6950-6961.

Maruyama, K., Yuda, T., Okamoto, A., Ishikura, C., Kojima, S., Iwatsuru, M., 1991. Effect of Molecular Weight in Amphipathic Polyethyleneglycol on Prolonging the Circulation Time of Large Unilamellar Liposomes. Chem. Pharm. Bull. 39, 1620-1622.

Maurer, N., Fenske, D.B., Cullis, P.R., 2001. Developments in liposomal drug delivery systems. Expert Opin. Biol. Ther. 1, 923-947.

Nawalany, K., Kozik, B., Kepczynski, M., Zapotoczny, S., Kumorek, M., Nowakowska, M., Jachimska, B., 2008. Properties of Polyethylene Glycol Supported Tetraarylporphyrin in Aqueous Solution and Its Interaction with Liposomal Membranes. J. Phys. Chem. B 112, 1223112239.

Nawalany, K., Rusin, A., Kępczyński, M., Mikhailov, A., Kramer-Marek, G., Śnietura, M., Połtowicz, J., Krawczyk, Z., Nowakowska. M., 2009. Comparison of photodynamic efficacy of 
Wilkosz et al. European Journal of Pharmaceutical Sciences 2017, 100, 116-125. http://dx.doi.org/10.1016/j.ejps.2017.01.007

tetraarylporphyrin pegylated or encapsulated in liposomes: In vitro studies. J. Photochem. Photobiol. B. 97, 8-17.

Nosé, S., 1984. A Unified Formulation of the Constant Temperature Molecular Dynamics Methods. J. Chem. Phys. 81, 511-519.

Nunes, C., Brezesinski, G., Pereira-Leite, C., Lima, J.L.F.C., Reis, S., Lucio, M., 2011. NSAIDs Interactions with Membranes: A Biophysical Approach. Langmuir 27, 10847-10858.

Parrinello, M., Rahman, A., 1981. Polymorphic transitions in single crystals: A new molecular dynamics method. J. Applied Phys. 52, 7182-7190.

Pereira-Leite, C., Nunes, C., Reis, S., 2013. Interaction of nonsteroidal anti-inflammatory drugs with membranes: In vitro assessment and relevance for their biological actions. Progress in Lipid Research 52, 571-584.

Rodríguez-Barrientos, D., Rojas-Hernández, A., Gutiérrez, A., Moya-Hernández, R., GómezBalderas, R., Ramírez-Silva, M.T., 2009. Determination of pKa values of tenoxicam from 1H NMR chemical shifts and of oxicams from electrophoretic mobilities (CZE) with the aid of programs SQUAD and HYPNMR. Talanta 80, 754-762.

Róg, T., Vattulainen, I., 2014. Cholesterol, sphingolipids, and glycolipids: What do we know about their role in raft-like membranes? Chem. Phys. Lipids 184, 82-104.

Shalaeva, M., Kenseth, J., Lombardo, F., Bastin, A., 2008. Measurement of Dissociation Constants (pKa Values) of Organic Compounds by Multiplexed Capillary Electrophoresis Using Aqueous and Cosolvent Buffers. J. Pharm. Sci. 97, 2581-2606. 
Wilkosz et al. European Journal of Pharmaceutical Sciences 2017, 100, 116-125. http://dx.doi.org/10.1016/j.ejps.2017.01.007

Shojaee, S.A., Rajaei, H., Hezave, A.Z., Lashkarbolooki, M., Esmaeilzadeh, F., 2013. Experimental measurement and correlation for solubility of piroxicam (a non-steroidal antiinflammatory drugs (NSAIDs)) in supercritical carbon dioxide. J. Supercrit. Fluid. 80, 38-43.

Stepniewski, M., Pasenkiewicz-Gierula, M., Róg, T., Danne, R., Orlowski, A., Karttunen, M., Urtti, A., Yliperttula, M., Vuorimaa, E., Bunker, A., 2011. Study of PEGylated Lipid Layers as a Model for PEGylated Liposome Surfaces: Molecular Dynamics Simulation and Langmuir Monolayer Studies. Langmuir 27, 7788-7798.

Torchilin, V.P., 2005. Recent Advances with Liposomes as Pharmaceutical Carriers. Nat. Rev. Drug. Discovery 4, 145-160.

Tsai, R.-S., Carrupt, P.-A., El Tayar, N., Giroud, Y., Andrade, P., Testa, B., Brée, F., Tillement, J.-P., 1993. Physicochemical and Structural Properties of Non-steroidal Antiinflammatory Oxicams. Helv. Chim. Acta. 76, 842-854.

Wytrwal, M., Bednar, J., Nowakowska, M., Wydro, P., Kepczynski, M., 2014. Interactions of serum with polyelectrolyte-stabilized liposomes:Cryo-TEM studies. Colloid Surfaces B. 120, $152-159$.

Yoon, M., Choi, H.N., Kwon, H.W., Park, K.H., 1988. Solvent Dependence of Absorption and Fluorescence Spectra of Piroxicam. A Possible Intramolecular Proton Transfer in the Excited State. Bull. Korean Chem. Soc. 9, 171-175. 